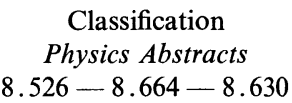

\title{
SATELLITE NMR STUDY OF THE DYNAMIC SUSCEPTIBILITY OF Fe IN Cu ABOVE AND BELOW $T_{\mathrm{K}}$
}

\author{
H. ALLOUL \\ Laboratoire de Physique des Solides $(*)$ \\ Université Paris-Sud, 91405 Orsay, France
}

(Reçu le 23 avril 1976, accepté le 9 juin 1976)

\begin{abstract}
Résumé. - Le temps de relaxation nucléaire $T_{1}$ des noyaux de ${ }^{63} \mathrm{Cu}$ premiers, seconds et troisièmes voisins du $\mathrm{Fe}$ dans les alliages $\mathrm{CuFe}$ a été mesuré dans $55 \mathrm{kG}$ pour $1,2 \mathrm{~K}<T<310 \mathrm{~K}$. Ces mesures permettent une détermination directe du taux de relaxation $\tau^{-1}$ de l'aimantation locale du Fe. $\tau^{-1}$ a une valeur constante pour $T \ll T_{\mathbf{K}}$, qui caractérise l'état de couplage fort du Fe et atteint une dépendance du type Korringa ( $\left.\tau^{-1} \alpha T\right)$ pour $T \gg T_{\mathrm{K}}$, en accord qualitatif avec les calculs théoriques sur l'effet Kondo.

Abstract. - NMR relaxation times $T_{1}$ of ${ }^{63} \mathrm{Cu}$ satellites corresponding to 1 st, 2 nd and 3rd nearest neighbours of $\mathrm{Fe}$ in $\mathrm{Cu}-\mathrm{Fe}$ dilute alloys have been measured in $55 \mathrm{kG}$ for $1.2 \mathrm{~K}<T<310 \mathrm{~K}$. These data allow a direct determination of the relaxation rate $\tau^{-1}$ of the local magnetization of $\mathrm{Fe}$. It is found to change from a constant value which characterizes the strong coupling state of Fe below $T_{\mathrm{K}}$ to a Korringa like behaviour $\tau^{-1} \alpha T$ for $T \gg T_{\mathrm{K}}$, as qualitatively expected from theoretical calculations on Kondo effect.
\end{abstract}

It has been clear for a long time that spin flip processes and consequently the fluctuations of the local magnetization play a major role in the establishment of the Kondo state of transition metal impurities in noble metal matrices [1]. It has thus been a great challenge for experimentalists to measure the frequency dependence of the local susceptibility, or at least its spectral width, which in ideal cases can be identified with the inverse of a spin autocorrelation time $\tau$ (or spin lattice relaxation time in spin resonance terminology). A great number of experimental techniques such as ESR [2], impurity [3] or host NMR $[4,5]$, Mössbauer effect [6], and neutron scattering [7] have been used to determine $\tau^{-1}$ in various characteristic systems. However up to now such experiments were only successful for limited ranges of temperature, either $T \ll T_{\mathrm{K}}$ or $T \gg T_{\mathrm{K}}$. In the classic $\mathrm{CuFe}$ Kondo system for which $T_{\mathrm{K}} \simeq 30 \mathrm{~K}$, recent nuclear spin lattice relaxation data for the near neighbour ${ }^{63} \mathrm{Cu}$ nuclei of Fe showed that for $T \ll T_{\mathrm{K}} \tau$ has a constant value $\tau^{-1} \sim k_{\mathrm{B}} T_{\mathrm{K}} / \hbar$ [5]. In this letter we present an extension of these measurements to room temperature, where for the first time the variation of $\tau^{-1}$ could be accurately followed from well below to well above $T_{\mathbf{K}}(\sim 30 \mathrm{~K})$.

Measurements have been carried out with pulse NMR techniques at about $60 \mathrm{MHz}$ on the three ${ }^{63} \mathrm{Cu}$ satellites A, M, B [8] which correspond to the 1st,

(*) Laboratoire associé au C.N.R.S. 2nd and 3rd nearest neighbours to Fe. Full details of the experimental technique will be reported elsewhere. Here the physical significance of the nuclear $T_{1}$ data which are summarized in figure 1 will be discussed. For satellite B data could be taken for a large range of concentration, showing that the measured $T_{1}$ values are characteristic of neighbours of isolated impurities. $T_{1}^{-1}$ is proportional to $T$ below $4.2 \mathrm{~K}$, goes through a maximum $(\sim 50 \mathrm{~K})$ then a minimum $(\sim 150 \mathrm{~K})$ and finally approaches the pure copper Korringa rate $\left(T_{1 \mathrm{~K}} T=1.28 \mathrm{~s} \mathrm{~K}\right)$ at higher temperature. The shorter relaxation times and lower sensitivity make $T_{1}$ measurements more difficult and less accurate for the first and second nearest neighbours.

It was shown in ref. [5] that, at low $T$, for various satellites, the relaxation is dominated by impurity induced relaxation due to isotropic hyperfine coupling with the transverse fluctuations of the impurity magnetization. For a shell of neighbours at distance $r$ from the impurity this extra relaxation rate is given by :

$$
\begin{aligned}
& {\left[T_{1}^{\mathrm{imp}}(r)\right]^{-1}=} \\
& \quad=2 \hbar^{-2} A_{\mathrm{s}}^{2}(r) k_{\mathrm{B}} T\left(g \mu_{\mathrm{B}}\right)^{-2} \operatorname{Im}\left\{\chi_{\mathrm{T}}\left(\omega_{\mathrm{n}}\right) / \omega_{\mathrm{n}}\right\},
\end{aligned}
$$

where $\chi_{\mathrm{T}}(\omega)$ is the transverse susceptibility of the impurity and $\omega_{n}$ the nuclear Larmor frequency. The isotropic transferred hyperfine coupling constant $A_{\mathrm{s}}(r)$ can be deduced from the differential Knight 


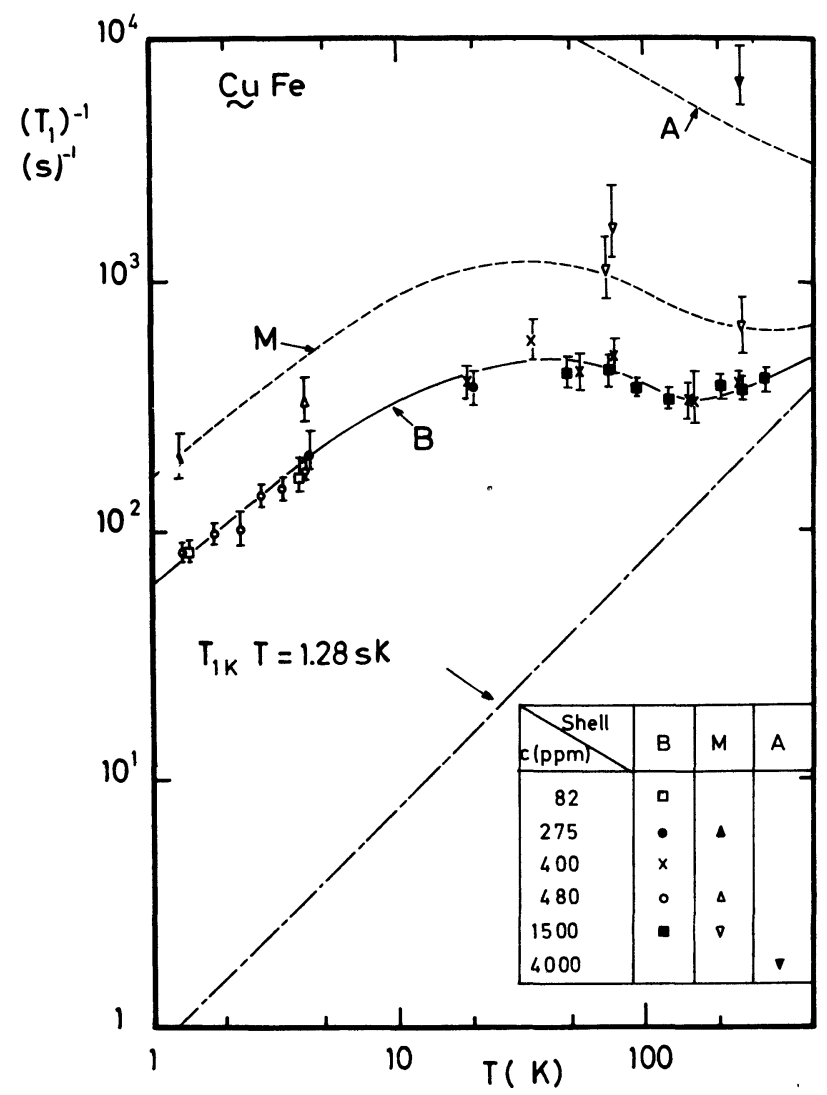

FIG. 1. - NMR relaxation rates of the 1st (A), 2nd (M) and 3rd (B) shells of neighbours of $\mathrm{Fe}$ in $\mathrm{Cu}$ measured near $55 \mathrm{kG}$. The impurity induced relaxation rate $\left(T_{1}^{\mathrm{imp}}\right)^{-1}$ is obtained by subtracting the Korringa rate $\left(T_{1 \mathrm{~K}}\right)^{-1}$ of pure $\mathrm{Cu}$. The dashed lines for satellites $\mathrm{A}$ and $\mathrm{M}$ are deduced from the solid line (which approximates the data for B), by assuming that $\left(T_{1}^{\text {imp }}\right)^{-1}$ scales with $(\Delta K)^{2}$.

shift of each shell of nuclei with respect to pure copper :

$$
\Delta K(r)=K(r)-K_{\text {pure }}=\left(\hbar^{2} \gamma_{\mathrm{e}} \gamma_{\mathrm{n}}\right)^{-1} A_{\mathrm{s}}(r) \chi_{\mathrm{L}},
$$

where $\chi_{\mathrm{L}}$ is the static longitudinal susceptibility of the impurity. Combining (1) and (2) yields

$$
\begin{aligned}
& {\left[T_{1}^{\mathrm{imp}}(r)\right]^{-1}=} \\
& \quad=2[\Delta K(r)]^{2} k_{\mathrm{B}} T \gamma_{\mathrm{n}}^{2} \operatorname{Im}\left\{\chi_{\mathrm{T}}\left(\omega_{\mathrm{n}}\right) / \omega_{\mathrm{n}} \chi_{\mathrm{L}}^{2}\right\} .
\end{aligned}
$$

The present results allow us to demonstrate that this relaxation process is still dominant at high $T$. Representing the data for satellite $B$ by the solid line drawn on figure 1 , we obtain estimates for the relaxation rates of satellites $\mathrm{A}$ and $\mathrm{M}$ (dashed lines) by assuming

$$
T_{1}^{-1}=\left(T_{1 \mathrm{~K}}\right)^{-1}+\left[T_{1}^{\mathrm{imp}}(r)\right]^{-1}
$$

with $\left[T_{1}^{\mathrm{imp}}(r)\right]^{-1}$ scaling as $\Delta K^{2}(r)$. The values $\Delta K\left(r_{\mathrm{A}}\right) / \Delta K\left(r_{\mathrm{B}}\right)=4.5$ and $\Delta K\left(r_{\mathrm{M}}\right) / \Delta K\left(r_{\mathrm{B}}\right)=1.63$ were taken from the data of ref. [9]. The good agreement between the dashed lines and the corresponding measured relaxation rates confirms that eq. (3) describes the relaxation. It should be noted that slight deviations from exact scaling with $\Delta K^{2}(r)$ might be associated with a small contribution of the anisotropic part of the hyperfine coupling to the nuclear relaxation [5]. In order to analyze the data further, we assume that the susceptibility is isotropic for $\omega \ll k_{\mathrm{B}} T_{\mathrm{K}}$, and that it can be characterized by a single correlation time $\tau$

$$
\chi_{\mathrm{T}}(\omega)=\chi_{\mathrm{L}} \frac{1+i \omega_{\mathrm{e}} \tau}{1-i\left(\omega-\omega_{\mathrm{e}}\right) \tau} .
$$

The value $\chi_{\mathrm{L}}=8.30 \times 10^{-26} \mathrm{emu} / \mathrm{atom}$ at $T=0$ [10], together with the experimental data for $T_{1}^{\text {imp }}$ and $\Delta K$ for satellite B allow an experimental determination of $\tau^{-1}$ from eq. (2), (3), (4). It can be seen from figure 2 that $\tau^{-1}$ departs from its constant value at $T=0$ and increases for $T \gg T_{\mathrm{K}}$, reaching a Korringa like dependence $\tau^{-1} \alpha T$ for $T \gg T_{\mathrm{K}}$. The resulting values for $\tau^{-1}$ are plotted in figure 2 , where the three data points obtained from inelastic neutron scattering experiments [7] are also shown. The quantitative agreement is surprisingly good.

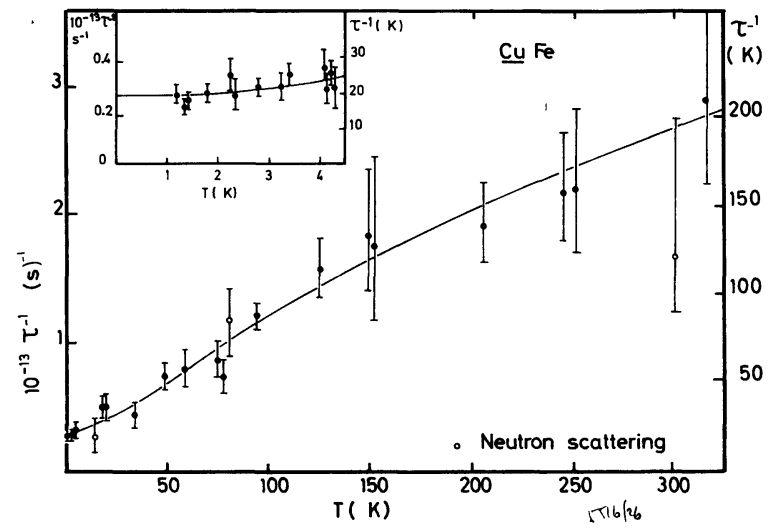

FIG. 2. - Experimental values of $\tau^{-1}$ deduced from the data of figure 1 . The three data points obtained from neutron scattering experiments [7] are in very good agreement with the present results. The solid line approximates the data points.

The two limiting temperature dependences could be expected from existing experimental data for $T \ll T_{\mathrm{K}}$ in weakly magnetic systems (Al-Mn, Au. V) [3] and for $T \gg T_{\mathrm{K}}$ in strongly magnetic systems (AgMn) [2]. They also appear in the theoretical results derived by Goetze and Schlottman in an approximate solution of the spin $1 / 2$ sd model [11]. In order to allow a quantitative analysis of the present data, we should rather consider theoretical results starting from the Anderson Hamiltonian, including orbital degeneracy of the impurity state. Using a perturbation expansion in $U / \pi \Delta$ ( $U$ intraatomic coulomb interaction, $\Delta$ virtual bound state width) according to the scheme developed by Yamada and Yosida [12], Shiba [13] showed that for $T \ll T_{\mathrm{K}}$ the simple relation :

$$
\lim _{\omega \rightarrow 0} \operatorname{Im} \chi_{\mathrm{T}}(\omega) / \omega \chi_{\mathrm{L}}^{2}=2 \pi /\left[\hbar \gamma_{\mathrm{e}}^{2}(2 l+1)\right]=\tau / \chi_{\mathrm{L}}
$$


holds irrespective of the value of $U / \pi \Delta$. This yields a Korringa relation for $T \ll T_{\mathrm{K}}$ and $\hbar \omega_{\mathrm{e}} \ll k_{\mathrm{B}} T_{\mathrm{K}}$

$$
\lim _{\omega_{\mathrm{n}} \rightarrow 0} T_{1} T(\Delta K)^{2}=K^{\mathrm{T}}(0) \&,
$$

where $S=\left(\hbar / 4 \pi k_{\mathrm{B}}\right)\left(\gamma_{\mathrm{e}} / \gamma_{\mathrm{n}}\right)^{2}$ is the classical Korringa constant for a free electron gas, and

$$
K^{\mathrm{T}}(0)=2 l+1=5 .
$$

We have already shown in ref. [5] that our low $T$ data is in very good agreement with eq. (6). In order to evaluate $\tau^{-1}$ in the high temperature limit, the case $U / \pi \Delta \gg 1$ will be considered, for which the Anderson and exchange Hamiltonians are equivalent but where the wave-vector dependence of the exchange is [14]

$$
J_{k k^{\prime}}=J_{\text {eff }} P_{l}(\cos \theta)
$$

where $P_{l}$ is the Legendre polynomial $(l=2)$ [15]. The corresponding Korringa relaxation rate for the impurity is [16] :

$$
\tau_{\mathrm{K}}^{-1}=\frac{\pi}{(2 l+1) \hbar}\left(J_{\mathrm{eff}} \rho\right)^{2} k_{\mathrm{B}} T,
$$

where $\rho$ is the density of states at the Fermi level. Assuming that $\chi_{\mathrm{L}}$ follows a Curie law (with spin $S$ ) yields also a Korringa relaxation like eq. (6) with a different constant :

$$
\varkappa^{\mathrm{T}}(\infty)=\frac{2 \pi^{2}}{3(2 l+1)} S(S+1)\left(J_{\text {eff }} \rho\right)^{2} .
$$

Then the quantity $\mathcal{K}(T)=T_{1} T \Delta K^{2} / \mathcal{S}$ is expected to change progressively from $\mathcal{K}^{\mathrm{T}}(0)$ to $\mathcal{K}^{\mathrm{T}}(\infty)$ when $T$ goes through the Kondo temperature. Our data for $\lim _{\omega \rightarrow 0} T_{1} T(\Delta K)^{2}$ in $\mathrm{CuFe}$ are given in figure 3, where it can be seen that, for $T<10 \mathrm{~K}, \mathcal{K}(T)=4.6 \pm 0.6$ is in very good agreement with $\pi^{\mathrm{T}}(0)$. But, surprisingly enough, the variation of $\pi(T)$ from 0 to $300 \mathrm{~K}$, if any, is very small (a least square fit to the data gives $\mathcal{K}(T)=3.9 \pm 0.5$ for $100 \mathrm{~K}<T<310 \mathrm{~K}$ ).

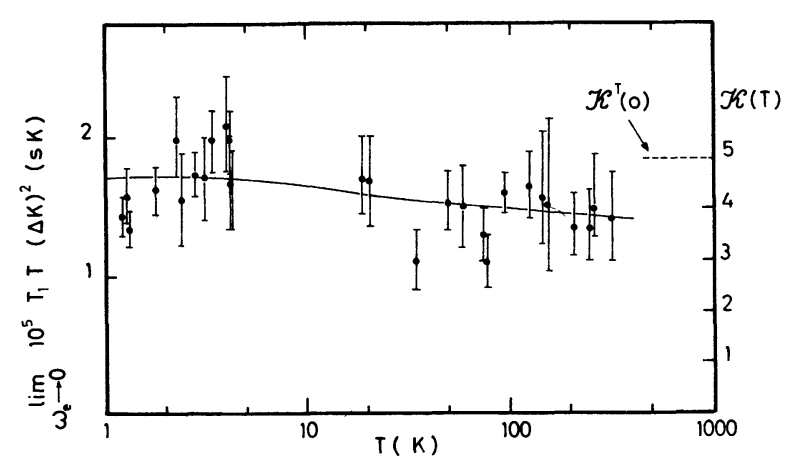

FIG. 3. - The zero frequency limit for the Korringa product $T_{1} T(\Delta K)^{2}$ can be seen to decrease only very slightly with tempe-

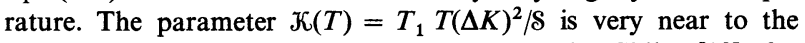
universal theoretical value $\kappa T(0)=5$ given by Shiba [12], for $T \ll T_{\mathrm{K}}$.
Whether this corresponds fortuitously to $\mathcal{K}^{\mathrm{T}}(\infty)$ being close to $\mathcal{K}^{\mathrm{T}}(0)$, or to $T \sim 300 \mathrm{~K}$ being still a low temperature for the limiting Korringa rate $\tau_{\mathbf{K}}^{-1}$ of eq. (6) to be reached requires an independent determination of $J_{\text {eff }} \rho$. An upper limit $J_{\text {eff }} \rho \lesssim 1$ can be deduced from eq. (8) assuming from figure 2 that $\hbar \tau_{\mathrm{K}}^{-1}=0.64 \quad k_{\mathrm{B}} T$. As no reliable theoretical calculation for the realistic case $S>1 / 2$ are available for $T \sim T_{\mathrm{K}}$, accurate determinations of $J_{\text {eff }} \rho$ are usually possible from comparisons of the data for various physical quantities with perturbation theory for $T \gg T_{\mathrm{K}}$. Such an approach is not possible in CuFe as measurements are only possible for $T \sim T_{\mathrm{K}}$ because of the high Kondo temperature. An approximate value for $J_{\text {eff }} \rho$ can be obtained from the expression of the Kondo temperature [14] :

$$
k_{\mathrm{B}} T_{\mathrm{K}}=D \exp \left[-(2 l+1) / J_{\text {eff }} \rho\right] \text {. }
$$

For $T_{\mathrm{K}}=30 \mathrm{~K}$, the deduced value for $J_{\text {eff }} \rho$ depends of the value taken for the bandwidth $D$ which is only known to have the same order of magnitude as the Fermi energy $\left(J_{\text {eff }} \rho=0.66\right.$ for $D=5 \mathrm{eV}$, $J_{\text {eff }} \rho=0.94$ for $D=0.5 \mathrm{eV}$ ). A better determination might be expected from the amplitude of the spin density oscillations :

$$
n(r)=-\frac{1}{4 \pi} J_{\text {eff }} \rho \frac{\cos 2 k_{\mathrm{F}} r}{r^{3}}\left\langle S_{z}\right\rangle .
$$

It is clear now on experimental grounds $[8,9,17]$ that eq. (11) holds at any temperature. Then, since the main influence of the Kondo effect on $n(r)$ appears in the variation of $\left\langle S_{z}\right\rangle$, comparison of the ${ }^{63} \mathrm{Cu}$ NMR linewidth data with the impurity magnetization should allow us to derive $J_{\text {eff }} \rho$. Unfortunately such an analysis requires an accurate knowledge of the $s$ hyperfine field constant of ${ }^{63} \mathrm{Cu}$ and of the exchange enhancement of the Pauli susceptibility of pure copper. The value $J_{\text {eff }} \rho=0.75$ was obtained from the data of Alloul et al. [17] but when analysed along the lines proposed by Walstedt and Walker [18], $J_{\text {eff }} \rho \sim 1$ is obtained. In any case for such large values of the effective exchange interaction, the use of the limiting exchange model of eq. (7) is questionable. For instance if $U / \pi \Delta$ is not large enough the value of $J_{\text {eff }} \rho$ which should be introduced in eq. (8), (9) and (10) are expected to be different functions of the parameters of the Anderson model. From this analysis it can only be concluded that $2 \lesssim K^{\mathrm{T}}(\infty) \lesssim 5$.

It should be mentioned at this point that the present results are somewhat different from those obtained on $\mathrm{Au} . \mathrm{V}$, for which $T_{1} T$ of ${ }^{51} \mathrm{~V}$ was found constant up to $T_{\mathrm{K}}(\sim 300 \mathrm{~K})$ [19], which corresponds to a decrease of $\mathcal{K}(T)$ of about a factor 4 from $T=0$ to $T \sim T_{\mathrm{K}}$, while $J_{\text {eff }} \rho$ might be expected to be larger than in $\mathrm{CuFe}$ (larger $T_{\mathrm{K}}$ ). These results (which incidentally also correspond to a decrease of $\tau^{-1}$ from $T=0$ to $T \sim T_{\mathrm{K}}$ ) seem somewhat difficult to understand, even qualitatively, when compared to the present ones. 
In conclusion new progress in the understanding of the dynamic properties of Kondo impurities has been achieved as these satellite NMR data allow one to study the change from the strong coupling regime to local moment behaviour of the dynamic susceptibility of $\mathrm{Fe}$ in $\mathrm{Cu}$. Nevertheless it cannot be decided presently from our data whether $\tau^{-1}$ is still enhanced with respect to the limiting Korringa law for $T \sim 10 T_{\mathrm{K}}$. Such a possibility is indeed suggested by the theoretical results of Götze and Schlottman [11], who indicate that many decades in temperature are necessary to reach the asymptotic behaviour, $\tau^{-1}$ being still enhanced by about a factor 10 for $T \sim 10 T_{\mathrm{K}}$. This possibility is apparently supported by some independent experimental results. In $\mathrm{Au} . \mathrm{Yb}$ a $\log T$ deviation from $\tau^{-1} \alpha T$ has been evidenced by Mössbauer effect measurements [6] for $0.5 \mathrm{~K}<T<5 \mathrm{~K}$, while $T_{\mathrm{K}}$ is known in this system to be less than $1 \mathrm{mK}[20,21]$. In CuMn $\left(T_{\mathrm{K}} \sim 10 \mathrm{mK}\right)$ the relaxation $\tau^{-1}$ measured at helium temperatures from ${ }^{63} \mathrm{Cu}$ NMR data [4] is much greater than that extrapolated from impurity NMR measurements at 1000 [22] and $300 \mathrm{~K}$ [23]. Similar NMR satellite experiments are presently being undertaken on CuMn and should allow a quantitative determination of the variation of $\tau^{-1}$ over a large range of temperatures above $T_{\mathbf{K}}$.

\section{References}

[1] A series of review articles devoted to Kondo effect can be found in Magnetism Vol. 5 GT Rado and H. Suhl, ed. (Academic Press New York) 1973.

[2] Davidov, D., Rettori, C., Orbach, R., Dixon, A. and CHOck, E. P., Phys. Rev. B 11 (1975) 3456.

[3] Narath, A., ref. [1], chap. 5.

[4] Alloul, H. and Bernier, P., J. Phys. F : Metal. Phys. 4 (1974) 870.

[5] Alloul, H., Phys. Rev. Lett. 35 (1975) 460.

[6] Gonzalez-Jimenez, F. and Imbert, P., Solid. State Commun. $13(1973) 85$

[7] Loewenhaupt, M. and Just, W., Phys. Lett. 53A (1975) 305.

[8] Boyce, J. B. and Slichter, C. P., Phys. Rev. Lett. 32 (1974) 61 and Phys. Rev. B 13 (1976) 379.

[9] Alloul H., AIP conference proceedings : Magnetism and Magnetic Materials Philadelphia (1975) to be published.

[10] Tholence, J. L. and Tournier, R., Phys. Rev. Lett. 25 (1970) 867.

[11] Götze, W. and Schlottmann, P., J. Low Temp. Phys. 16 (1974) 87.

[12] Yamada, K., Prog. Theor. Phys. 54 (1975) 316 and ref. therein.
[13] Shiba, H., Prog. Theor. Phys. 54 (1975) 967.

[14] Blandin, A., J. Appl. Phys. 39 (1968) 1285.

[15] In this particular case of $\mathrm{Cu}-\mathrm{Fe}$, which has a high Kondo temperature, the direct exchange term can be neglected with respect to the sd mixing.

[16] Davidov, D., Maki, K., Orbach, R., Rettori, C. and CHоск, E. P., Solid State Commun. 12 (1973) 621.

[17] Alloul, H., Darville, J. and Bernier, P., J. Phys. F. Metal Phys. 4 (1974) 2050.

[18] Walstedt, R. E. and WalkeR, L. R., Phys. Rev. B 11 (1975) 3280 .

[19] Kazama, S., Kume, K., Mizuno, K. and Mizoguchi, K., Phys. Lett. 42A (1972) 141.

[20] Benoit, A., Flouquet, J. and Sanchez, J., Phys. Rev. B 9 (1974) 1092.

[21] Frossati, G., Mignot, J. M., Thoulouze, D. and TourNIER, R., Phys. Rev. Lett. 36 (1976) 203.

[22] Walstedt, R. E. and Warren, W. W., Phys. Rev. Lett. 31 (1973) 365 .

[23] Kume, K. and Mizoguchi, K., Solid State Commun. 16 (1975) 675 . 